Available online at GSC Online Press Directory

GSC Biological and Pharmaceutical Sciences

e-ISSN: 2581-3250, CODEN (USA): GBPSC2

Journal homepage: https://www.gsconlinepress.com/journals/gscbps

(RESEARCH ARTICLE)

\title{
COVID-19 wavelet coherence data for some Gulf countries
}

\author{
Vyacheslav Lyashenko ${ }^{1}$, Omer Ibrahim Abdallah Mohammed ${ }^{2}$ and Asaad Mohammed Ahmed Babker ${ }^{3, *}$ \\ ${ }^{1}$ Department of Informatics, Kharkiv National University of Radio Electronics, Ukraine. \\ 2 Deportment of Hematology, College of Medical Laboratory Sciences, Omdurman Islamic University, Khartoum, Sudan. \\ ${ }^{3}$ Deportment of Hematology, College of Medical Laboratory Sciences, University of Science and Technology, Omdurman, \\ Sudan.
}

Publication history: Received on 22 April 2020; revised on 10 May 2020; accepted on 12 May 2020

Article DOI: https://doi.org/10.30574/gscbps.2020.11.2.0130

\begin{abstract}
Coronaviruses are one of the most dangerous forms of viruses. The development of the COVID-19 pandemic takes the form of a potential threat to all of humanity. This is due to the fact that coronaviruses have high pathogenicity, the ability to overcome human immunity. There are also difficulties in treating diseases that are associated with coronavirus. To solve such issues, it is important to obtain reliable statistical data, as well as conduct a comprehensive analysis of the data that are currently received. Based on this, the paper considers the possibility of analyzing data on the development of the COVID-19 pandemic based on wavelet analysis approaches. The wavelet coherence method was used as the main approach for statistical data analysis. The consistency of the results for different countries of the Persian Gulf is shown. Based on the analysis of the depth of cross-references between the studied data series, the most significant time periods were obtained until patients recover or die. Some explanation is given for the patterns that arise for individual Gulf countries. The data obtained can be used in the fight against the pandemic COVID-19, understanding the dynamics of its development.
\end{abstract}

Keywords: Viruses; COVID-19; Pandemic; Wavelet analysis; Wavelet coherence

\section{Introduction}

Viruses, infections are one of the components of human life [1, 2]. The unpredictability and specificity of the effects of viruses and infections on humans, the ability to mutate complicates the fight against these sources of potential danger [3].

Currently, the global challenge facing humanity is the development of the COVID-19 pandemic. As of April 3, 2020, according to the World Health Organization, the total number of infected people with the COVID-19 coronavirus is 972 303, and the total number of deaths is 50321 (according to Situation report - 74. Coronavirus disease 2019 (COVID19). 3 April 2020. https://www.who.int/docs/default-source/coronaviruse/situation-reports/20200403-sitrep-74covid-19-mp.pdf?sfvrsn=4e043d03_12). All this happened in just 3 months of the development of the COVID-19 pandemic.

Therefore, the efforts of all mankind are aimed at overcoming the consequences of the action of the coronavirus COVID19. Among such efforts, an understanding of the available general statistics that describe the development of the COVID19 pandemic should be highlighted. One of the tasks of analyzing statistical data is the search for certain patterns, the possibility of explaining such patterns. Ultimately, this allows us to understand the nature of the development processes of the COVID-19 pandemic. Thus, the main goal of this study is to analyze general statistical data and identify possible patterns of the development of the COVID-19 pandemic.

\footnotetext{
* Corresponding author: Asaad Mohammed Ahmed Babker
}

Copyright (C) 2020 Author(s) retain the copyright of this article. This article is published under the terms of the Creative Commons Attribution Liscense 4.0. 


\section{Material and methods}

\subsection{A small review of research publications}

Various statistical methods are used to study the development processes of the COVID-19 pandemic.

A. L. Ziff and R. M. Ziff consider the development of the COVID-19 pandemic based on fractal kinetics [4]. This makes it possible to obtain new assessments for the development of the COVID-19 pandemic. At the same time, the authors compare their research with the works of other authors, pointing to their shortcomings. This makes it possible to obtain new models and more accurate results.

A. Brandenburg examines various distribution laws to explain mortality processes during the development of the COVID-19 pandemic [5]. A. Brandenburg compares the exponential and quadratic model of the increase in deaths from COVID-19. The author gives his explanations for various models, which allows a better understanding of the situation that arises in the process of obtaining new statistics data.

Q. Yang, C. Yi, A. Vajdi, L. W. Cohnstaedt, H. Wu, X. Guo and C. M. Scoglio consider different distribution models of COVID19 [6]. These models are based on Markovian processes and non-Markovian processes. Such a comparison allows a better understanding of the distribution path of COVID-19, taking the necessary measures to respond to possible scenarios of the development of the COVID-19 pandemic. In [6], it was shown that non-Markovian models provide a better explanation of the distribution processes of COVID-19.

S. L. Chang, N. Harding, C. Zachreson, O. M. Cliff and M. Prokopenko consider the computer model of COVID-19 distribution [7]. This model takes into account the age of infected people, the speed of spread of the disease, and measures to isolate people [7]. This allows you to evaluate the time period for controlling the distribution of COVID-19.

Particular attention is paid to the selection of the distribution model of the COVID-19 epidemic. To do this, different authors analyze various distribution laws, which allows us to describe the primary statistical data. The choice of data distribution laws is based on accurate statistical criteria and estimates between different data groups. In this case, the possible distribution laws are determined by daily data. An example of such research is the works [8-10].

S. Deb and M. Majumdar consider the distribution model of COVID-19, which is based on the quadratic trend of the spread of the disease [11]. The paper shows that such a model allows us to develop measures to counter the pandemic.

L. Jia, K. Li, Y. Jiang and X. Guo compare forecast models for analyzing COVID-19 distribution processes such as logistic model, Bertalanffy model, and Gompertz model [12]. This comparison allows a comprehensive analysis of processes of distribution COVID-19, build appropriate forecasting models.

Thus, different methods and approaches are used to analyze the of distribution processes of COVID-19, research primary statistics. This allows you to draw appropriate conclusions and make decisions that are necessary. At the same time, there is a need to expand such research. This will help to understand all the processes that occur as a result of the development of the COVID-19 pandemic. One of the directions for the development of relevant studies is a mutual analysis of existing primary statistics (total number of infected COVID-19, number of deaths, number of recovered). The solution to this problem is possible through the use of wavelet ideology, which has shown good results in the study of medical data [13-15].

\subsection{Wavelet coherency as a data analysis tool}

The ideology of wavelets allows the analysis of various processes where the data are presented in the form of a time series [16]. Such an analysis is based on the search for changes in the values of the series being investigated [15]. Among the methods of wavelet ideology, wavelet coherence should be distinguished [16, 17].Wavelet coherence allows cross analysis for the data that is being investigated. Such an analysis can be done for different depths of the data series that are being investigated. This allows us to justify the conditions for complete data consistency between the data series that are being studied.

To determine the values of wavelet coherence, we consider the values of cross wavelet spectra $W_{x y}(g, q)$ for two data series $g(t)$ and $q(t)$ ( $\mathrm{x}$ is the variable that displays the data number in the series under investigation, $\mathrm{y}$ is the 
variable that displays the depth of cross-links for a time series, $t$ - time interval being investigated). The general formula has the following form $[16,17]$ :

$$
R^{2}(g, q)=\frac{\left|V\left(k^{-1} W_{x y}(g, q)\right)\right|}{V\left(k^{-1}\left|W_{x}(g, q)\right|^{2}\right) V\left(k^{-1}\left|W_{y}(g, q)\right|^{2}\right)},
$$

where: $\mathrm{V}$ is a smoothing operator, $0 \leq R^{2}(g, q) \leq 1$. If the values $R^{2}(g, q)$ tend to zero, then we have a weak correlation. Otherwise, we have a strong correlation $[16,17]$.

\subsection{Data for analysis}

Among the Gulf countries, this study examines: Iran, Iraq, Kuwait, Oman, Qatar and Saudi Arabia. These countries have been selected in view of the different trends in the development of the COVID-19 pandemic, the various numbers of people and the size of their territories. For other Gulf countries, the results that we get can be interpolated taking into account their features. General data for these countries is publicly available on websites:

https://www.worldometers.info/coronavirus/,

https://www.who.int/emergencies/diseases/novel-coronavirus-2019/situation-reports,

https://github.com/datasets/covid-19,

https://datahub.io/core/covid-19.

We are considering the period 01/22/2020 - 02/04/2020. Among the data that we are considering, there is - total number of confirmed cases of COVID-19, total number of recovered, total number of deaths. At the same time, it should be noted that for different countries the data are presented for different periods. Therefore Fig. 1 illustrates a logarithmic dependence of the data in time.

From the data in Fig. 1 we see the different dynamics of the development of the COVID-19 pandemic. We can also observe completely different dynamics of deaths due to the spread of COVID-19 in each country that we analyze. There is an explanation for this - each country differs in the level of medicine, the level of response to the development of the situation, the level of reliability of statistical data.

Thus, in order to understand the development processes of the COVID-19 pandemic, we consider the corresponding estimates of wavelet coherence. 
Vyacheslav et al. / GSC Biological and Pharmaceutical Sciences, 2020, 11(02), 166-174

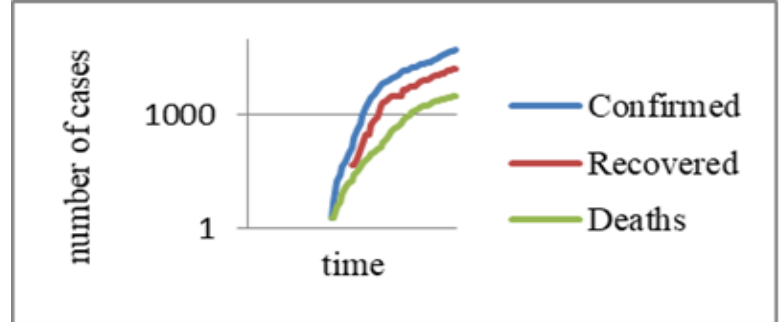

a) Iran

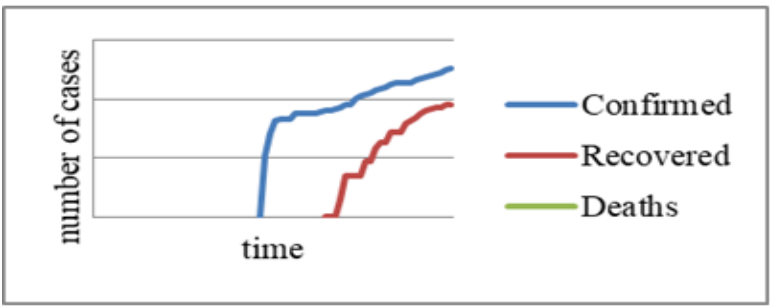

c) Kuwait

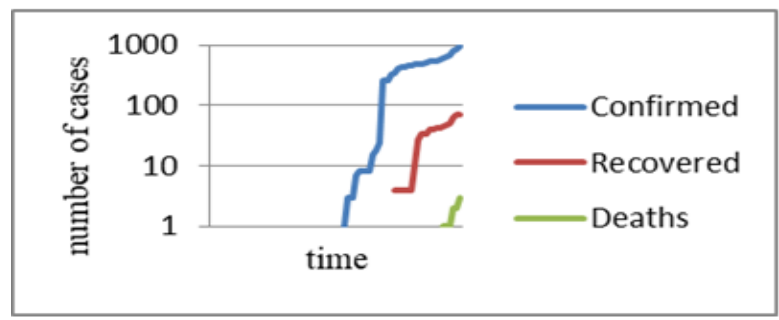

e) Qatar

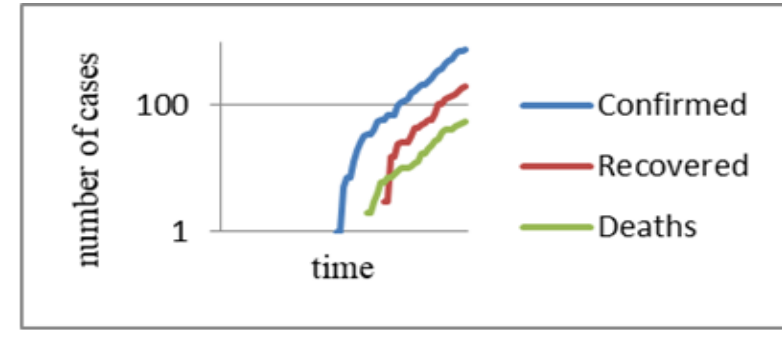

b) Iraq

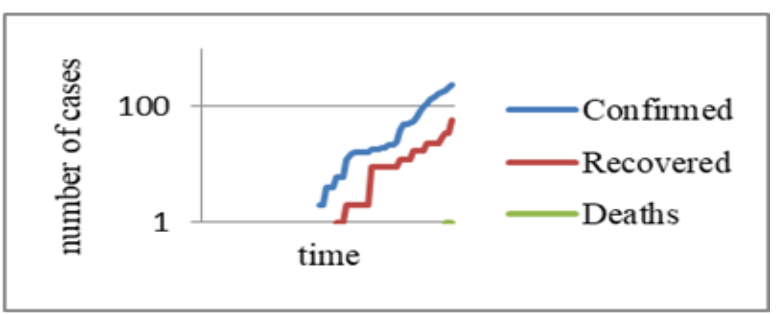

d) Oman

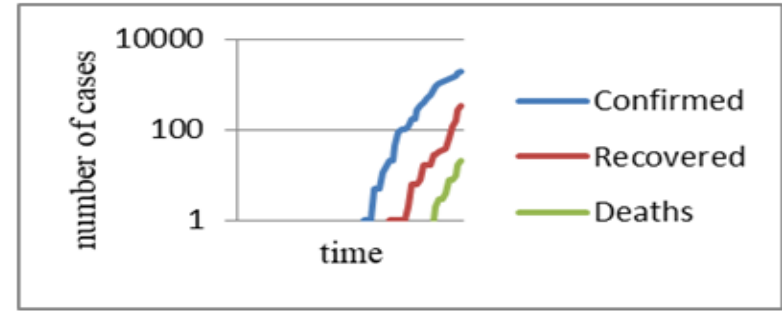

f) Saudi Arabia

Figure 1 Dynamics of the development of the COVID-19 pandemic in the Gulf countries (logarithmic dependence shown)

\section{Results and discussion}

Coronaviruses are one of the dangerous sources of human infection. Such a source can be fatal. At the same time, there are certain difficulties in the treatment of diseases that are associated with coronavirus. To solve such issues, it is important to obtain reliable statistics. Equally important is the analysis of the data that are available. Moreover, the necessary condition for the use of such data is the ability to explain the existing relationships between the various parameters that describe the dynamics of the pandemic [18].

We will examine estimates of wavelet coherence between the total number of confirmed cases of COVID-19 and the total number of recovered, as well as between the total number of confirmed cases of COVID-19 and the total number of deaths. This will determine the depth of periods of general consistency between the series of data that are being investigated. As a result, this data can be estimates that characterize the onset of outcomes (recovery or death) after infection with the COVID-19 coronavirus.

It should also be noted that the source data has different lengths. The defining data series is a data series that shows the total number of confirmed cases of COVID-19. Therefore, all other data series will be compared with a data series that shows the total number of confirmed cases of COVID-19. In some cases, we will not consider the relationship between some data series. This will apply to cases where the data series described by the total number of deaths is not large enough. In this case, we do not consider such data for Kuwait, Oman, Qatar (see Fig. 1).

In Fig. 2 - Fig. 7 presents the results for estimating the wavelet coherence for each of the Gulf countries (in accordance with the data in Fig. 1 and the remarks indicated above).

In Fig. 2 - Fig. 7, the abscissa axis indicates the time period, which is considered for each country separately. The measurement interval is one day. The ordinate axis indicates the cross-reference depth scale. The dashed white line limits the region of reliable 
values of wavelet coherence (with a confidence level of at least 0.95). Each figure (right) also shows a scale for analyzing the significance of wavelet coherence data (from 0 to 1). Such data for clarity have color values.

In Fig. 2 shows the wavelet coherence values for data from Iran. These data are for the period 02.19.2020 - 02.04.2020. Fig. 2a shows the relationship between total number of confirmed cases of COVID-19 and total number of recovered. Fig. $2 b$ shows the relationship between total number of confirmed cases of COVID-19 and total number of deaths.

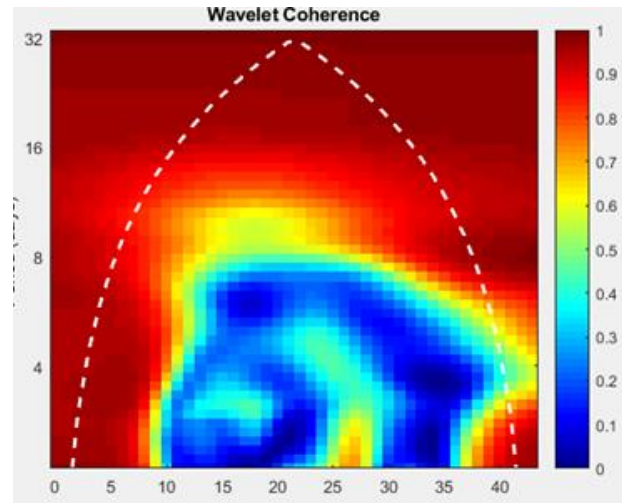

a)

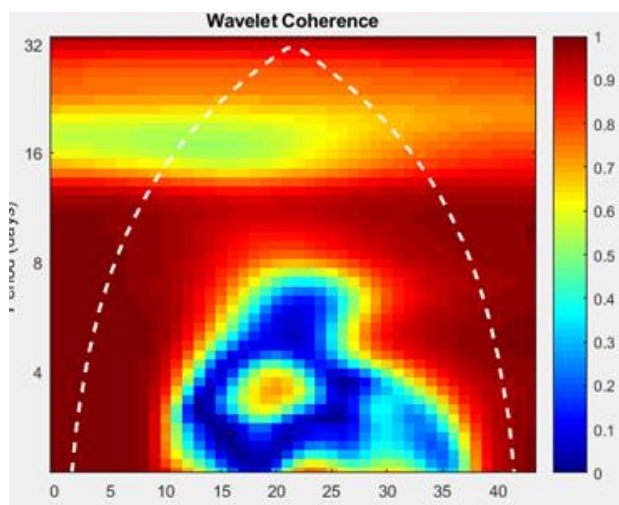

b)

Figure 2 Wavelet coherence values for data from Iran

From the data in Fig. 2 shows that the recovery or the onset of death has a small depth of cross-references for the beginning of the period that is being studied and the end of such a period. To start the period, the explanation of this fact is the lack of complete data. For the end of the period, an explanation of this fact is a continuation of the development of the COVID-19 pandemic. For other time periods, the smallest depth of the respective cross-reference is at 4 to 8 days. This may also be evidence of a lack of complete and reliable data. The reason for such comments is the fact that a possible recovery (depending on the severity of the disease) by the median number of days from the onset of symptoms is possible after 14 days of hospitalization [19]. At the same time, the median number of days before the onset of death may be minimal (depending on the length of hospitalization and the time of detection of infection) [20,21].

In Fig. 3 shows the wavelet coherence values for data from Iraq. These data are for the period 24.02.2020-02.04.2020. Fig. 3a shows the relationship between total number of confirmed cases of COVID-19 and total number of recovered. Fig. $3 \mathrm{~b}$ shows the relationship between total number of confirmed cases of COVID-19 and total number of deaths.

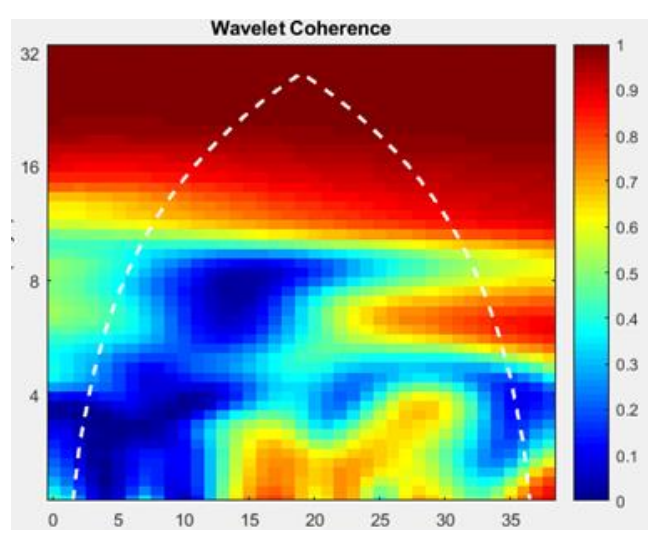

a)

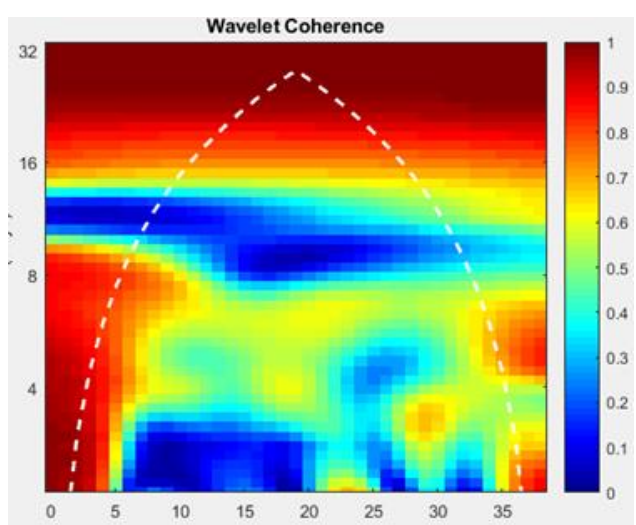

b)

Figure 3 Wavelet coherence values for data from Iraq 
Fig. 3 show, that the most significant and stable cross-reference depth in terms of the possible recovery or death of patients with COVID-19 is 12-16 days. This is already in better agreement with the data of studies that were carried out in $[19,10]$.

In Fig. 4 shows the wavelet coherence values for data from Kuwait. These data are for the period 24.02.2020-02.04.2020. Fig. 4 shows the relationship between total number of confirmed cases of COVID-19 and total number of recovered.

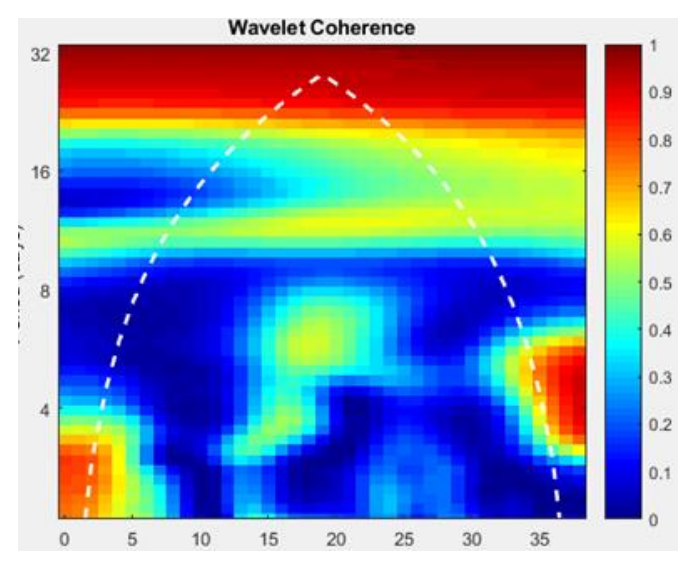

Figure 4 Wavelet coherence values between total number of confirmed cases of COVID-19 and total number of recovered for data from Kuwait

From the data in Fig. 4 shows that the cross-reference depth for the wavelet coherence estimates between the total number of confirmed cases of COVID-19 and the total number of recovered for data from Kuwait is 24 days. At the same time, you can see less significant data in 4-8 days. Given the fact that no patients who died in the time period that we are considering, we can talk about the effectiveness of healthcare services or the absence of severe disease COVID-19.

In Fig. 5 shows the wavelet coherence values for data from Oman. These data are for the period 24.02.2020-02.04.2020. Fig. 5 shows the relationship between total number of confirmed cases of COVID-19 and total number of recovered.

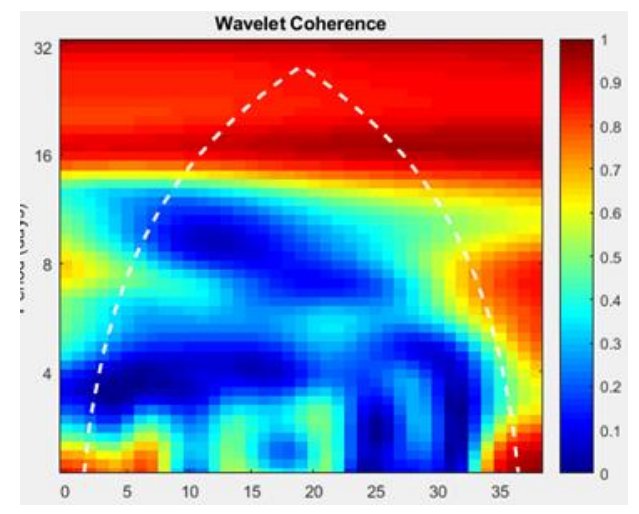

Figure 5 Wavelet coherence values between total number of confirmed cases of COVID-19 and total number of recovered for data from Oman

Data Fig. 5 is identical to the data in Fig. 4. Therefore, identical conclusions can be drawn. At the same time, it should be noted that the minimum cross-reference depth for wavelet coherence estimates between the total number of confirmed cases of COVID-19 and the total number of recovered for data from Oman is 15-16 days.

In Fig. 6 shows the wavelet coherence values for data from Qatar. These data are for the period 02.29.2020 - 02.04.2020. Fig. 6 shows the relationship between total number of confirmed cases of COVID-19 and total number of recovered. 


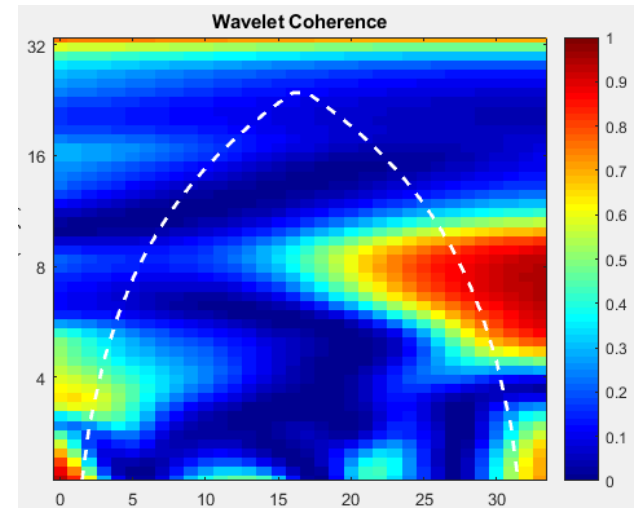

Figure 6 Wavelet coherence values between total number of confirmed cases of COVID-19 and total number of recovered for data from Qatar

Fig. 6 do not provide significant data on the existence of stable relationships between total number of confirmed cases of COVID-19 and total number of recovered.

In Fig. 7 shows wavelet coherence values for data from Saudi Arabia. These data are for the period 02.03.202002.04.2020. Fig. 7a shows the relationship between total number of confirmed cases of COVID-19 and total number of recovered. Fig. 7b shows the relationship between total number of confirmed cases of COVID-19 and total number of deaths.

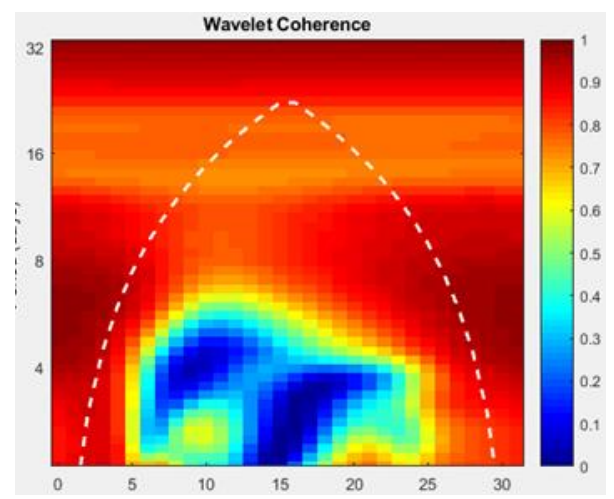

a)

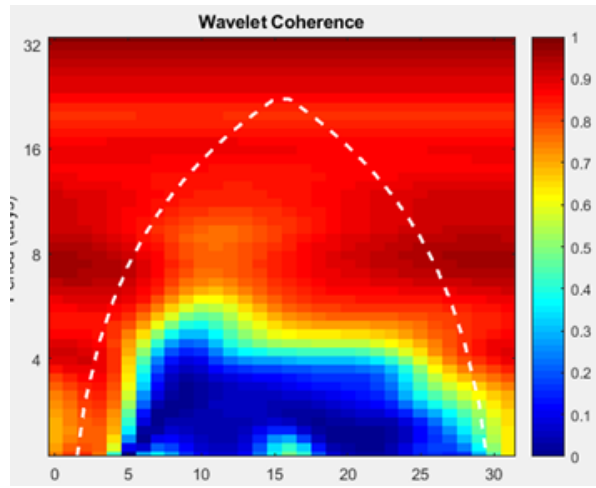

b)

Figure 7 Wavelet coherence values for data from Saudi Arabia

Data Fig. 7 are similar to the data in Fig. 2. Therefore, the conclusions for the data in Fig. 7 are identical to the findings for the data in Fig. 2.

In Fig. 8 shows the wavelet coherence values for data from all the Gulf countries that we are exploring. These data are for the period 01.29.2020 - 02.04.2020. Fig. 8a shows the relationship between total number of confirmed cases of COVID-19 and total number of recovered. Fig. 8b shows the relationship between total number of confirmed cases of COVID-19 and total number of deaths. 


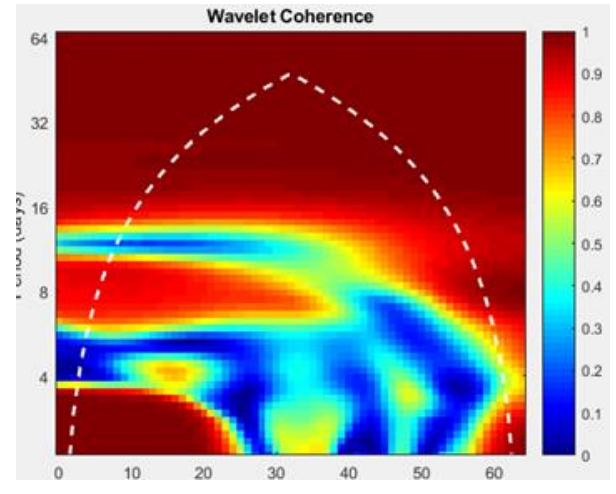

a)

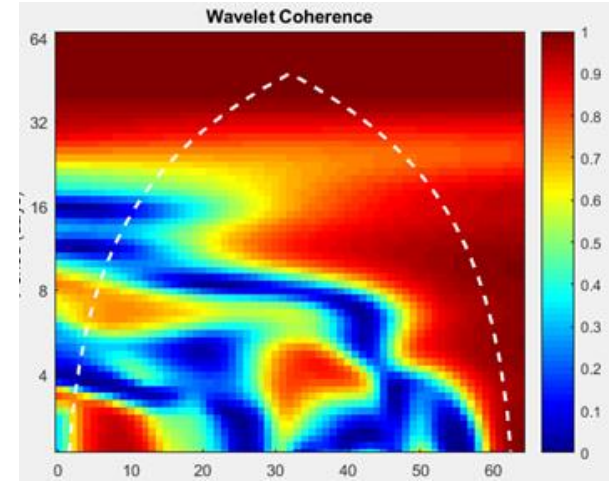

b)

Figure 8 Wavelet coherence values for data from Gulf countries

Data Fig. 8 summarize the results that were obtained previously. We can see that the most significant and stable crossreference depth for wavelet coherence estimates between the total number of confirmed cases of COVID-19 and the total number of recovered for data from the Persian Gulf countries starts from 16 days.

The depth of cross-references for estimating the wavelet coherence between the total number of confirmed cases of COVID-19 and the total number of deaths in the first half of the study period is not strictly defined. For the second half of the study period, this depth varies from 4 days and is the most stable from 12 days and above. The explanation for this may be a small number of deaths for most Gulf countries, with the exception of Iran. At the same time, it should be noted that for most Gulf countries, mortality of patients from COVID-19 is observed in the last 10-12 days, from the studied time period.

Therefore, the data in Fig. 8 can be used to assess the development of the COVID-19 pandemic in each Gulf country. We can use data such as boundary criteria.

\section{Conclusion}

We analyzed general statistics that describe the dynamics of the COVID-19 pandemic. Some Gulf countries were selected as a separate study region. For analysis, we used one of the approaches of the wavelet ideology - the wavelet coherence method. This method makes it possible to assess the degree of influence between the data series that are being investigated. The basis of this analysis is the study of the depth of cross-references between data series. We obtained results that explain some points in the dynamics of the COVID-19 pandemic in the Gulf countries. The results of wavelet coherence for the generalized data of the Gulf countries were also obtained. It is shown that such data can be used as boundary criteria.

\section{Compliance with ethical standards}

\section{Acknowledgments}

The authors are grateful to the Department of Informatics Kharkiv National University of Radio Electronics, the Deportment of Hematology College of Medical Laboratory Sciences Omdurman Islamic University and the Deportment of Hematology College of Medical Laboratory Sciences, University of Science and Technology Omdurman.

\section{Disclosure of conflict of interest}

The authors declare that there is no conflict of interest.

\section{References}

[1] Rook G, Bäckhed F, Levin BR, McFall-Ngai MJ and McLean AR. (2017). Evolution, human-microbe interactions, and life history plasticity. The Lancet, 390(10093), 521-530.

[2] Brodin P and Davis MM. (2017). Human immune system variation. Nature reviews immunology, 17(1), 21-29. 
[3] Wang Y, Yu L, Kong X and Sun L. (2017). Application of nanodiagnostics in point-of-care tests for infectious diseases. International journal of nanomedicine, 12, 4789-4803.

[4] Ziff AL and Ziff RM. (2020). Fractal kinetics of COVID-19 pandemic. medRxiv.

[5] Brandenburg A. (2020). Quadratic growth during the 2019 novel coronavirus epidemic. arXiv preprint arXiv:2002, 03638.

[6] Yang Q, Yi C, Vajdi A, Cohnstaedt LW, Wu H, Guo X and Scoglio CM. (2020). Short-term forecasts and long-term mitigation evaluations for the COVID-19 epidemic in Hubei Province, China. medRxiv.

[7] Chang SL, Harding N, Zachreson C, Cliff OM and Prokopenko M. (2020). Modelling transmission and control of the COVID-19 pandemic in Australia. arXiv preprint arXiv, 2003, 10218.

[8] Zhang S, Diao M, Yu W, Pei L, Lin Z and Chen D. (2020). Estimation of the reproductive number of Novel Coronavirus (COVID-19) and the probable outbreak size on the Diamond Princess cruise ship: A data-driven analysis. International journal of infectious diseases, 93, 201-204.

[9] Shi $\mathrm{H}$ et. al. (2020). Radiological findings from 81 patients with COVID-19 pneumonia in Wuhan, China: a descriptive study. The Lancet Infectious Diseases, 20, 425-434.

[10] Jung SM et. al. (2020). Real-time estimation of the risk of death from novel coronavirus (COVID-19) infection: Inference using exported cases. Journal of clinical medicine, 9(2), 523.

[11] Deb S and Majumdar M. (2020). A time series method to analyze incidence pattern and estimate reproduction number of COVID-19. arXiv preprint arXiv, 2003, 10655.

[12] Jia L, Li K, Jiang Y and Guo X. (2020). Prediction and analysis of Coronavirus Disease 2019. arXiv preprint arXiv, 2003, 05447.

[13] Abd Elgadir A, Babker AM, Osman AL, Ismail M and Lyashenko V. (2019). New Approach for Analysis the Correlation of Some Oxidative Markers in Type 2 Diabetes Mellitus by Data Wavelet Analysis. Indian Journal of Public Health, 10(11), 2449-2455.

[14] Ali WM et al. (2019). Wavelet coherence as a tool for visualizing the relationship between glomerular filtration rate and renal artery blood flow velocity. International Journal of Emerging Trends in Engineering Research, 7(12), 818-823.

[15] Lyashenko VV, Babker AMAA and Kobylin OA. (2016). The methodology of wavelet analysis as a tool for cytology preparations image processing. Cukurova Medical Journal, 41(3), 453-463.

[16] Torrence C and Webster PJ. (1999). Interdecadal changes in the ENSO-monsoon system. Journal of climate, 12(8), 2679-2690.

[17] Ng EK and Chan JC. (2012). Geophysical applications of partial wavelet coherence and multiple wavelet coherence. Journal of Atmospheric and Oceanic Technology, 29(12), 1845-1853.

[18] Lyashenko V, Eltayib Siddig MS and Asaad MB. (2020). Some data of wavelet analysis on the overall dynamics of the dead and recovered during infection COVID-19. World Journal of Advanced Research and Reviews, 6(2), 17 23.

[19] Ronco C, Navalesi P and Vincent JL. (2020). Coronavirus epidemic: preparing for extracorporeal organ support in intensive care. The Lancet Respiratory Medicine, 8(3), 240-241.

[20] Benavides EM. (2020). Robust predictive model for Carriers, Infections and Recoveries (CIR): predicting death rates for CoVid-19 in Spain. arXiv preprint arXiv, 2003, 13890.

[21] Kumar P et al. (2020). Forecasting the dynamics of COVID-19 Pandemic in Top 15 countries in April 2020 through ARIMA Model with Machine Learning Approach. medRxiv.

\section{How to cite this article}

Lyashenko V, Omer Ibrahim AM and Asaad Mohammed AB. (2020). COVID-19 wavelet coherence data for some Gulf countries. GSC Biological and Pharmaceutical Sciences, 11(2), 166-174. 